\title{
Understanding and Overcoming Proximity Effects in Multi-Spot Two-Photon Direct Laser Writing
}

\section{Authors}

Caroline Arnoux, ${ }^{1 *}$ Luis A. Pérez-Covarrubias, ${ }^{2}$ Alexandre Khaldi, ${ }^{2}$ Quentin Carlier, ${ }^{2}$ Patrice L.Baldeck, ${ }^{1}$ Kevin Heggarty, ${ }^{2 *}$ Akos Banyasz ${ }^{1}$ and Cyrille Monnereau ${ }^{1}$

\author{
Affiliations \\ ${ }^{1}$ Univ. Lyon, ENS Lyon, CNRS, Université Lyon 1, Laboratoire de Chimie, UMR 5182, 46 Allée \\ d'Italie, 69364 Lyon, France. \\ 2 IMT-Atlantique, Optics Department, Technopole Brest-Iroise, 29285, BREST, France. \\ c.caroline.arnoux@gmail.com \\ kevin.heggarty@imt-atlantique.fr
}

\begin{abstract}
Although additive manufacturing using multi-photon direct laser writing is nowadays considered as a major tool in the fabrication of future nano/micro-objects and optical components, it is currently limited by the low throughput of the writing process. To circumvent this issue, massive parallelization of the write process is a very promising avenue. However, simultaneous writing of structures in close spatial proximity generates fabrication artefacts, collectively referred to as "proximity effects", which strongly limit the accessible structure resolution. In this work, we systematically investigate the experimental parameters that influence these effects using specifically designed $\mathrm{N} \times \mathrm{N}$ spot diffractive optical elements. Through computer simulations, we show that these effects can be modeled remarkably successfully simply by taking Point Spread Function overlap and diffusion processes into account. We illustrate the usefulness of the concept by designing a parallel write approach giving access to periodic structures with short inter-object distances while very largely overcoming proximity effects.
\end{abstract}

\section{Keywords}

Two-photon, Direct Laser Writing, proximity effect, radical polymerization, parallel microfabrication

\section{Introduction}

Additive manufacturing has attracted increasing interest due to the large number of applications that can be addressed, from aeronautics to medical devices and optics [1]. Large surfaces with micron and sub-micron patterns such as lens arrays for sensors or micro-needle arrays for drug delivery are part of the structures that can be built by two-photon induced polymerization (TPP) also called direct laser writing (DLW) [1-3]. Through focusing a laser source into a small volume of liquid resin with a high numerical aperture objective, this technique enables the fabrication of complex and highly-resolved three-dimensional

microstructures.

However, this technique suffers from long plot times for large samples (up to days for objects with dimensions in the millimeter range), which currently slows down its entry into the industrial applications market [1-3]. To overcome this major limitation, different strategies have been implemented such as the use of galvanometer scanners to increase the scanning speed, whether or not in association with multi-focus spot setups. Multi-foci can be provided by microlens arrays (MLA), spatial light modulators (SLM), ultrafast random-access digital micromirror devices (DMD) or diffractive optical elements (DOE) [4-13]. In all these strategies, massive parallelization increases throughput by increasing the number of focal spots used simultaneously so that large areas or arrays of periodic structures can be written in the same time frame traditionally used to fabricate a single structure with a single beam.

Unfortunately, this parallelization can have a detrimental effect on the uniformity of the repeated structures due to the so-called "proximity effects". Polymerization appears to depend on the local photochemical environment and the intensity distribution further away from the desired focal spots. Thus, reducing the distance between structures proves to be more challenging than reducing the feature sizes due to structure broadening [14-16] and sporadic connections [15,17-19] that arise between them. When simultaneously exposing several spots, this effect is exacerbated and different local TPP thresholds are 
observed, for example, at the center and in the corner of a write spot array. This effect is attributed to diffusion phenomena $[15,16,20,21]$ and has already been observed for conventional single photon polymerization [22] as well as in DLW, both with low one-photon absorption (LOPA) [23] and two-photon absorption $[15,16]$. It has even been already taken advantage of to improve surface smoothness [24]. Although it has occasionally been highlighted in articles dealing with DLW, proposing tracks to correct it mainly with dynamic irradiation power control [15,16,22,23,25-27], its precise and quantitative characterization is scarce and has, to our knowledge, never been reported in detail for parallel plotting. It has also recently been identified as a key difficulty that must be overcome to enable significant parallelization speed improvements through the use of large numbers of closely spaced write spots [13].

In this paper, we introduce an experimental study to characterize the dependence of the proximity effects encountered in parallel microfabrication based on the use of DOEs in various experimental conditions. By proximity effects we more precisely mean: any local changes in the 2PP threshold linked to the presence of other light spots in the vicinity of a studied light spot (spatial proximity effects) or to any previous exposure of the resist - whether leading to polymerization or not - by spots scanned across the same plot area (temporal proximity effects). This approach has been combined with a phenomenological computer model allowing us to demonstrate the effect of point spread function (PSF) overlap and diffusion processes, partially quantifying the range of the effect under specific conditions. We thus highlight three distinct regimes depending on the write spot separation: i/ small inter-structure spacings where light spot overlap effects outside the focal plane (Talbot-like effects) make any attempt of parallel twophoton DLW extremely challenging; ii/ intermediate spacings in which diffusion (for example of radicals) plays an important role in creating "crosstalk" between exposed spots (proximity effects) and undesired overpolymerization that can, however, be overcome; iii/ large spacings where no particular difficulties arise. Ultimately, we show that it is possible to circumvent these proximity effects using larger separations between spots.

This work provides a better understanding of the role of diffusion in proximity effects, and offers new insights for massively parallelized two-photon DLW generally considered today to be one of the most promising approaches for high-throughput, high-resolution manufacturing [13].

\subsection{DOE preparation}

The spot array DOEs used to parallelise the write process were all designed using a modified three-stage Iterative Fourier Transform Algorithm (IFTA) [28-31]. DOEs with different target images containing different numbers of output spots and different spots separations (corresponding to different DOE spatial periods and hence diffraction angles) were calculated and fabricated. Typical inter-spot diffraction angles ranged from $1^{\circ}$ to $0.01^{\circ}$. The DOEs were all binary phase elements, etched into a layer (thickness $\sim 400 \mathrm{~nm}$ ) of spin-coated S1805 photoresist (MicroChem) on $1.1 \mathrm{~mm}$ thick float glass substrates using a custom-built, massively parallel-write photoplotter [30,31]. Typical DOE experimental diffraction efficiencies of $70-75 \%$ were observed. When appropriate, target output spot patterns with a deliberately weakened central spot were chosen to compensate for increased zeroth order spot power resulting from inevitable DOE fabrication process limitations and hence obtain increased experimental array spot power uniformity. Occasionally, over-correction led to a slight underexposure of the central spot.

\subsection{Microfabrication}

Resin preparation: 1,10-decanediol diacrylate (DDA) was purchased from TCI, dipentaerythritol penta/hexaacrylate (DPPHA) from Merck, unstabilized Ormocomp® from Micro Resist Technology GmbH, and 4-methoxyphenol (MEHQ) from Acros Organics. All reagents were used without further purification. Three resins were used in this study based on the use of a photoinitiator (PI) previously reported by our team [32,33]: PR_organic (DPPHA/DDA/V-Shape, weight ratio: 79.2/19.8/1, includes $c a .600$ ppm MEHQ as stabilizer/polymerization inhibitor), PR_hybrid (PI-free Ormocomp/V-Shape, weight ratio: 99.5/0.5, stabilizer free) and stabilized PR_hybrid (PI-free Ormocomp/VShape, weight ratio: 99.5/0.5 + 700 ppm MEHQ). In the first formulation PR_organic (DPPHA/DDA/V-Shape, weight ratio: 79.2/19.8/1), V-Shape was mixed with 1,10-decanediol diacrylate (DDA) and stirred for 30 min at room temperature and then dipentaerythritol penta-/hexa-acrylate (DPPHA) was added without additional solvent (PR_organic). The solution was stirred mechanically for $1 \mathrm{~min}$ and then magnetically for 30 min.

For the second resin PR_hybrid (PI-free Ormocomp/VShape, weight ratio: 99.5/0.5), the PI was mixed with a special PI-free Ormocomp resin and dichloromethane. Dichloromethane was allowed to evaporate overnight 


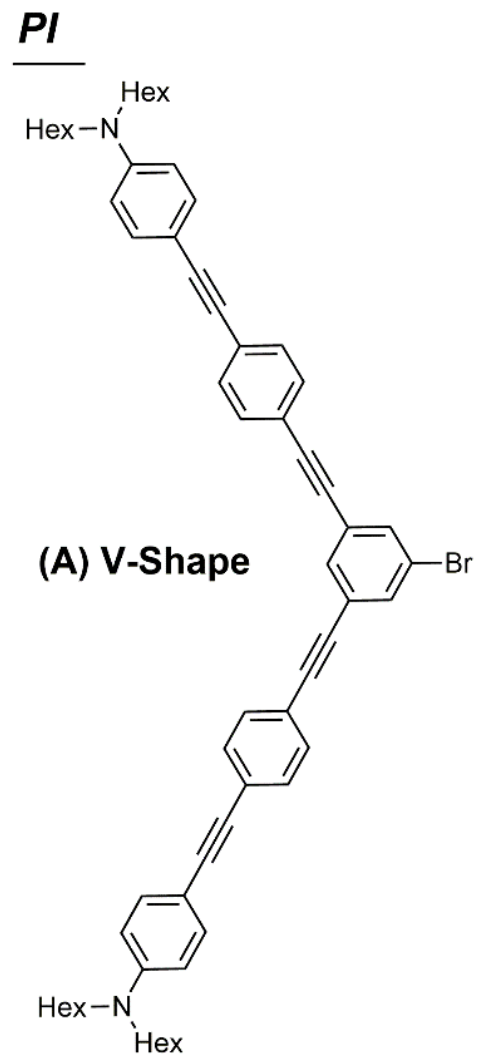

\section{Monomers}<smiles>C=CC(=O)OCCCCCCCCCCCCCC(=O)OCCCC</smiles>

(B) DDA

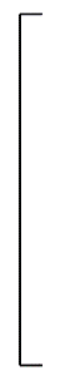<smiles>C=CC(=O)OCC(CO)(COCC(COC(=O)C=C)(COC(=O)C=C)COC(=O)C=C)COC(=O)C=C</smiles><smiles>C=CC(=O)OCC(COCC(COC(=O)C=C)(COC(=O)C=C)COC(=O)C=C)(COC(=O)C=C)COC(=O)C=C</smiles>

(C) DPPHA<smiles>C=CC(=O)OCC(CC)(COC(=O)C=C)COC(=O)CCSCCC[Si](C)(OC)OC</smiles>

(D) Ormocomp inorganic-organic precursor

Fig. 1. Chemical structures of the PI and the monomers used in this study: (A) V-Shape $[32,33]$; (B) 1,10decanediol diacrylate DDA; (C) dipentaerythritol penta/hexa-acrylate DPPHA; (D) Ormocomp is an organicinorganic hybrid network based on the precursor mentioned herein.

under stirring. Homogeneous resist was obtained without filtering.

The third formulation PR_hybrid stabilized with 700 ppm MEHQ was made following the same procedure as PR_hybrid but adding MEHQ along with V-Shape. Molecular structures are shown in Fig. 1.

Fabrication and setup: 3D microfabrication was performed on a Microlight3D printer $\mu$ FAB-3D based on a Zeiss Axiovert 200 inverted microscope equipped with a XYZ piezo nanomanipulator allowing the translation of the sample relative to the laser focal point, and a CMOS camera mounted behind a dichroic mirror to monitor the polymerization process. The laser module includes a microchip self-Q-switched frequency-doubled Nd:YAG laser $(532 \mathrm{~nm}, 560 \mathrm{ps}$ pulses, $11.7 \mathrm{kHz}$ repetition rate, $11.5 \mathrm{~mW}$ maximum average power at the entrance of the microscope objective). Average laser powers were measured at the entrance pupil of the objective on a standard photodiode power sensor (S120VC, Thorlabs). The incident beam was focused with one of two different objectives $(\times 100$, NA 1.40, oil immersion, Zeiss Plan-APOCHROMAT and $\times 40$, NA 0.95 , dry, Zeiss Plan-APOCHROMAT). The laser power, the displacement of the sample relative to the objective and the scanning speed were computercontrolled via LITHOS software. The DOEs were introduced at the entrance of the dichroic mirror cube in the reflector turret of the inverted microscope. The samples consist of resin drops disposed on borosilicate coverslips (170 $\pm 5 \mu \mathrm{m}$ thick). After the fabrication process, the microstructures were finally obtained by washing away the unreacted monomer using acetone (two successive 10-minute baths).

Microstructure characterization: Samples were metallized with a $5 \mathrm{~nm}$ thick gold coating via vacuum deposition with Leica EM ACE600 sputter coater and observed by Scanning Electron Microscopy (SEM) on a Zeiss Supra 55VP SEM operating at a tension of $5 \mathrm{kV}$, using a top view $\left(0^{\circ}\right)$ or a $45^{\circ}$ view.

\subsection{Phenomenological digital simulations of the polymerization and diffusion processes}

The parallelised write process, photo-chemical polymerization interactions and associated diffusion phenomena were modelled via digital simulations written in MATLAB, based on Point Spread Functions (PSF) calculated using the PSF Generator [34] software with the Gibson-Lanni model. The PSF represents the 3D light intensity distribution of the laser beam in the objective focal plane. Our software models the DOE 


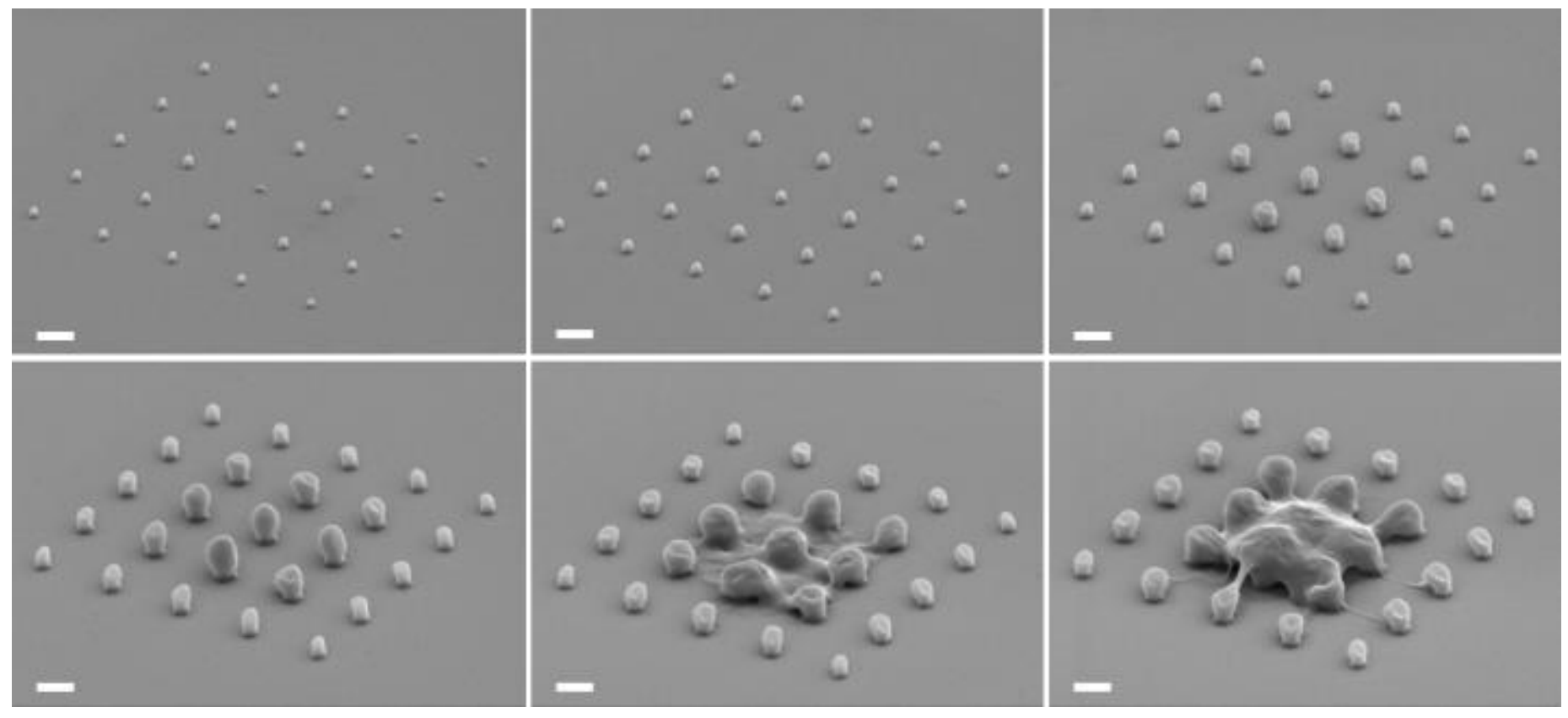

Fig. 2. Illustration of the proximity effects on a voxel array manufactured in PR_Hybrid Ormocomp/VShape with a $5 \times 5$ spot DOE with an inter-spot distance of $2.22 \mu \mathrm{m}$ with increasing total laser power (top line, from left to right: $1.9-2.6-3.5 \mathrm{~mW}$; bottom line, from left to right: $4.0-4.4-4.8 \mathrm{~mW}$ ). Exposure time is constant $(100 \mathrm{~ms})$. Scale bar: $1 \mu \mathrm{m}$.

generated light spot array as a 3D matrix of PSFs (Fig.

S5).

The write process was modelled as the sequential, cumulative addition of several PSF arrays, one for each laser pulse, with a translation of the array between pulses as required to model the beam scanning process. Proximity effects between light spots were allowed for by introducing a diffusion step (convolution by a 3D Gaussian function) between each laser pulse. Several diffusion processes are possible, notably molecular diffusion such as radical or inhibitor diffusion (but heat energy brought by the laser and exothermic chemical interactions could also be involved). Our digital modelling of this diffusion is simplistic and purely phenomenological, making no a priori assumptions about the underlying physico-chemical mechanisms: we simply assume a diffusion process takes place, and calculations show that the characteristic lifetime of the diffusing species are compatible with values reported for carbon centred radical species. Polymerization was modelled by thresholding the cumulated light energy dose during the process, applying non-linear functions as required to represent multi-photon processes. A sampling grid of $200 \mathrm{~nm}$ was chosen as a compromise between maintaining manageable computing loads and modelling the PSF functions with sufficient fidelity. Supplementary details regarding mathematical simulation and parameters are featured as SI.

\section{Results and discussion}

\subsection{A qualitative illustration of proximity effects in parallel write}

An introductory illustration of the proximity effects can be seen in Fig2. where one of the resists involved in the study (PR_hybrid Ormocomp/V-Shape, see experimental section for details about the composition) is exposed with an array of irradiation beams $(5 \times 5$ spot array with a $2.22 \mu \mathrm{m}$ inter-spot distance) of increasing irradiation intensity. At low intensity, all irradiated spots present a similar polymerization pattern, slight variations being due to non-uniformities in the diffraction pattern resulting from imperfections in DOE fabrication (see experimental section). Upon increasing the intensity, it becomes very apparent that the pattern progressively changes, with an increased polymerization efficiency in the center in comparison with the periphery (Fig 2.). This evolution corresponds to increasing overlap and crosstalk in the exposed area, and can be influenced by many parameters that we will explore in the rest of this article.

\subsection{Influence of the write spot separation}

Fig 3A features two structures fabricated with spot arrays with an equal number of spots but different spot separations. The observed differences clearly illustrate the influence of the spot separation: small spacing increases the inhomogeneity even with short exposure times. Thus, it is not possible to achieve homogeneous polymerization of each voxel structure using an $11 \times 11$ spot DOE with an inter-spot distance of $0.62 \mu \mathrm{m}$ 
A)

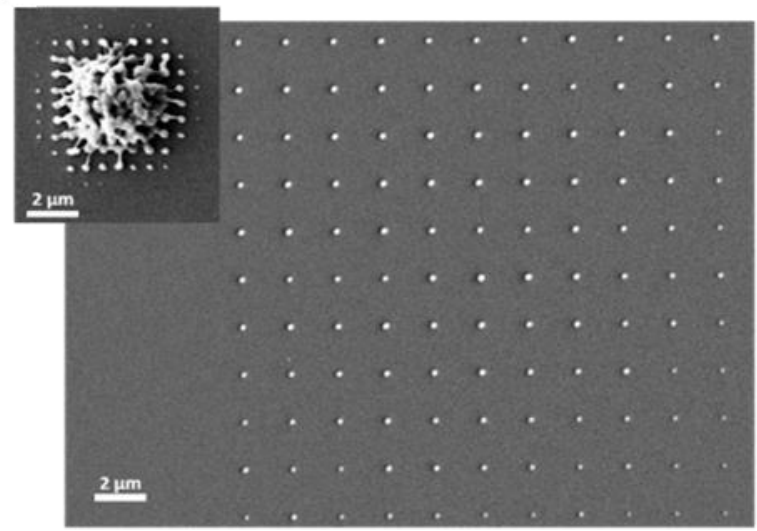

B)

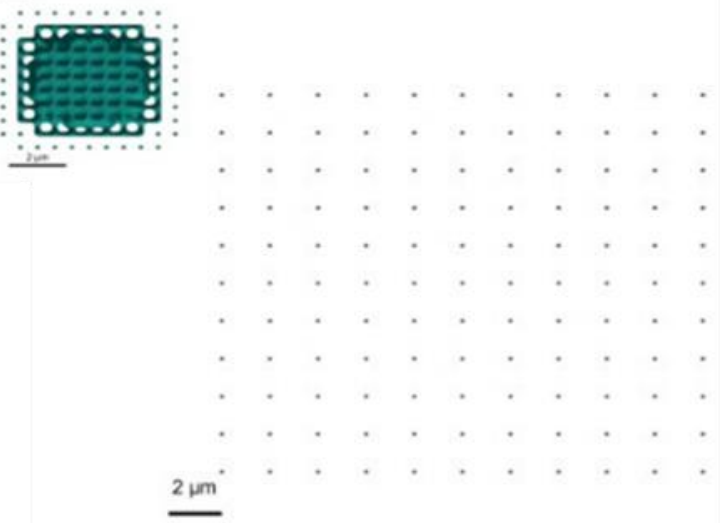

Fig. 3. (A) Voxel arrays manufactured in the PR_organic resin with two $11 \times 11$ spot DOEs with inter-spot distances of $0.62 \mu \mathrm{m}$ (insert top left) and $1.85 \mu \mathrm{m}$ (right) at $5.4 \mathrm{~mW}$ total average incident laser power on the DOE using the 100x objective. The exposure times were $50 \mathrm{~ms}$ and 5 $s$ for the $0.62 \mu \mathrm{m}$ and $1.85 \mu \mathrm{m}$ spacings respectively. (B) Results of the digital simulation (including diffusion) of the parallel DLW process, showing the effect of spot distance, for voxel arrays manufactured in the PR_organic resin with two $11 \times 11$ spot DOEs with diffusion. The inter-spot distances are $0.6 \mu \mathrm{m}$ (insert top left) and $1.8 \mu \mathrm{m}$ (right).

whereas the analogue structure is easily obtained with an $11 \times 11$ spot DOE with a three times greater inter-spot distance $(1.85 \mu \mathrm{m})$. Note that the array of voxel structures with the $0.62 \mu \mathrm{m}$ spacing observed in Fig. 3A, was obtained with hundred-times shorter exposure time compared to that used for the fabrication with the $1.85 \mu \mathrm{m}$ spaced DOE. The central overpolymerization observed with the closely spaced spots is accompanied by an underpolymerization of the peripheral structures, so that some of the voxel structures in the last two rows were washed away during the development step. This behaviour supports the hypothesis that the proximity of the spots during simultaneous exposure contributes to reducing the overall polymerization threshold of each structure written in parallel, all the more so as we move

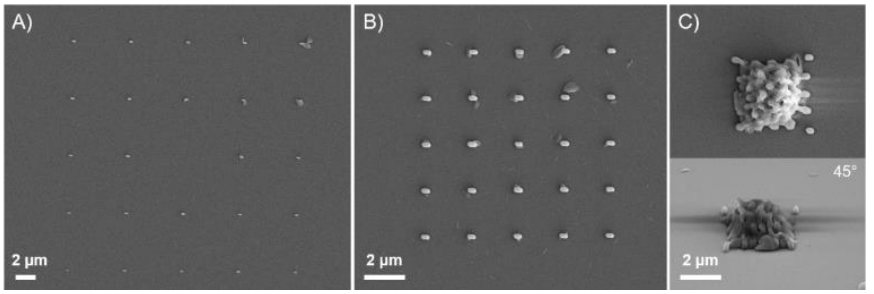

Fig. 4. Arrays of voxels manufactured with three $5 \times 5$ spot DOEs at decreasing inter-spot distance. Spacing from left to right: (A) 5.55, (B) 2.22, (C) $0.89 \mu \mathrm{m}$. $P_{\text {total }}=4.0 \mathrm{~mW}$, exposure time: $50 \mathrm{~ms}$. Resin: PR_organic. 100x objective.

closer to the center of the structure array. At small write spot spacings, this results in a non-uniform spatial profile of the polymerization threshold and a decrease of each threshold compared to sequential single-beam writing $[27,35]$.

In order to test this hypothesis, we modelled these experimental conditions in our digital simulations by using 585 and 58500 laser pulses to take into account the experimental exposure times $(50 \mathrm{~ms}$ and $5 \mathrm{~s}$, respectively) and laser repetition rate $(11.7 \mathrm{kHz})$. Fig. 3B shows the results of these simulations for the 100x NA 1.40 objective PSF with write spot spacings of 0.6 $\mu \mathrm{m}$ (left) and $1.8 \mu \mathrm{m}$ (right), the approximation in the choice of the simulated distances being imposed by the characteristics of our sampling grid (see experimental section).

We first simulated the overlapping of the PSFs without introducing diffusion effects into the numerical model (Fig. S1). In this case, no overpolymerized areas were observed in the simulated structures. In contrast, when adding diffusion and decay parameters in the simulations, an overall result that is remarkably similar to the experimentally obtained structures was achieved (Fig. 3B). Despite our digital model's simplicity, this result indicates that the simulation of the parallel write process as a PSF overlap and basic diffusion process appears to be a reasonably accurate description.

The influence of inter-spot separation in the polymerization threshold is even more clearly demonstrated in Fig. 4 showing microfabricated voxel structure arrays obtained with different spacings but in otherwise identical exposure conditions. 


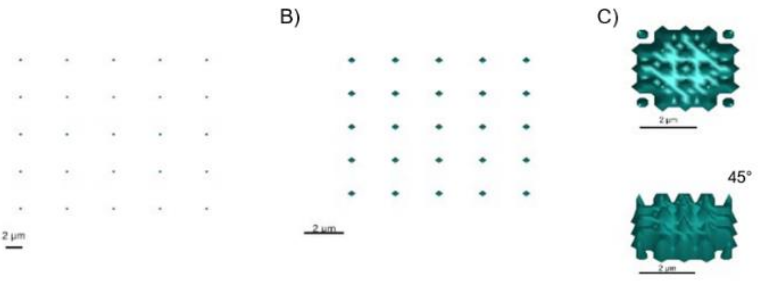

Fig. 5. Results of the digital simulation of the parallel write process showing of the effect of spot separation for $5 \times 5$ arrays. (A) $5.6 \mu \mathrm{m}$, (B) $2.2 \mu \mathrm{m}$ and (C) 0.8 $\mu \mathrm{m}$. Total number of laser pulses was 585 in each case.

While reducing the inter-spot distance from ca. $5.6 \mu \mathrm{m}$ to $2.2 \mu \mathrm{m}$, the voxel array remains reasonably homogeneous, except for the slightly over-corrected central spot; indeed, the array is more uniform, indicating a possible use of the proximity effects as a corrective parameter to the overcorrection of zeroorder. However, at a $0.9 \mu \mathrm{m}$ inter-spot distance, the voxel structures at the edges appear clearly smaller than those that are closer to the center and uncontrolled polymerization has occurred at the center, resulting in structures with ill-controlled height and the merging of individual voxels into an undefined intricate structure.

Again, the experimental results were confronted with numerical simulations using the same model as described above. The results of the corresponding simulations in which $5 \times 5$ spot arrays with different separations (5.6 $\mu \mathrm{m}, 2.2 \mu \mathrm{m}$ and $0.8 \mu \mathrm{m})$ were used, can be seen in Fig. 5. Based on the exposure time and laser repetition rate, 585 laser pulses were considered in each case and the diffusion parameters were kept constant compared to the previous simulation since the experiments were conducted with the same resin (see Table S1). A decay factor of 0.995 (assuming exponential decay) corresponds to a species half-life of $0.012 \mathrm{~s}$, in good agreement with previously reported values for short-lived radical species [36]. As in the previous experiment, we can see a strong similarity between the simulated structures and the experimental results. A noticeable difference is the height of the simulated structures which tends to saturate ("flat top") as a consequence of the limited $\mathrm{Z}$ range of the simulations (computing load limitations).
In the experiments at small inter-spot distances, these typical overpolymerized structures, which seem to spread vertically above the focal plane, could result from "out-of-plane" overlap of the PSFs. As the light focused by the microscope objective converges to form the array of spots in the focal plane, the local light energy density increases towards the write spot locations. If the spots in the array are sufficiently separated, the energy density will be too low outside the focal plane for overlap between the light fields from the different light spots to reach the polymerization threshold. However, when the spots are closer together, at planes close to the focal plane, the converging light may concentrate sufficiently for overlap to produce polymerization (see Fig. S2).

This generation of out of focus plane "hot spots" bears some resemblance to the well-known Talbot effect which can lead to periodic repetitions of an image of the spot pattern at regular distances away from the focal plane, the first layer being shifted by half the period of the focal spot pattern [37]. The Talbot effect has already been used for patterning and 3D printing applications such as displacement Talbot lithography $[38,39]$ or proximity field nanopatterning [40]. However, the Talbot effect generally occurs when all the spots in a regular array have the same light field phase. This is not the case with the DOEs used here since they generate spots with random light field phases and are therefore likely to produce random interference patterns due to overlap in out of focus planes.

Such effects are also clearly seen in Fig. S3, showing structures obtained when using a small spot separation write-spot array that was scanned to write " $L$ " shaped motifs: increasing the incident light power results in the progressive formation of a multi-layered periodic structure along the $\mathrm{Z}$ axis. Although clearly distinct from the diffusion-related proximity effect illustrated in the previous and following examples, it also results in undesired polymerization peaking at the center of the generated motif, and should be taken into account in parallel fabrication especially when generating periodic structures with short inter-voxel distances.

\subsection{Influence of the Objective Numerical Aperture}

Besides inter-spot distance which partly depends on the magnification of the objective, the numerical aperture of the objective used for the fabrication is another important parameter that influences proximity effects, because it will affect light distribution and inter-spot overlaps of the PSF functions. In order to address this issue, parallel fabrication using a 0.95 NA (Zeiss $40 \times$ 


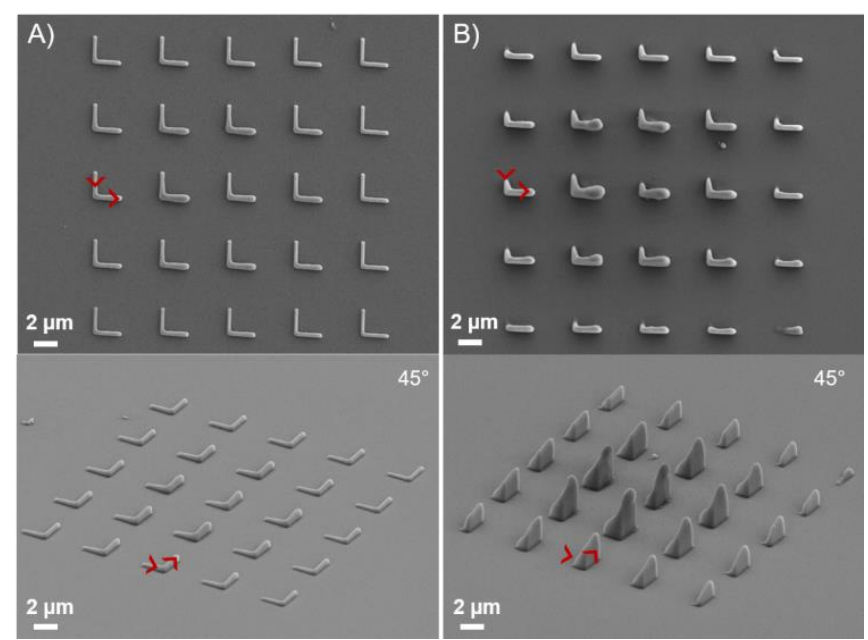

Fig. 6. Influence of the microscope objective on the uniformity of $5 \times 5$ "L" networks $(2 \mu \mathrm{m}$ per arm) separated by $5.55 \mu \mathrm{m}$. (A) Objective 100x (NA 1.40), $P_{\text {total }}=3 \mathrm{~mW}, \mathrm{v}=2.4 \mu \mathrm{m} \cdot \mathrm{s}^{-1}$. (B) Objective 40× (NA 0.95), $P_{\text {total }}=9.0 \mathrm{~mW}, \mathrm{v}=2.4$ $\mu \mathrm{m} . \mathrm{s}^{-1}$. Resin: PR_hybrid. For each array, top (top) and $45^{\circ}$ (bottom) views are shown and the writing direction is marked by red arrows. The structures of network (A) are fairly uniform while those of network (B) show important disparities.

Apochromat) and a 1.40 NA (Zeiss 100× Apochromat) microscope objective were compared.

Due to the different magnification of the objectives, the spot spacing obtained with the $40 \times$ objective is 2.5 times that obtained with the $100 \times$ objective for the same DOE. To enable reliable comparison of both objectives at same inter spot distance in the final fabricated array of structures, we used two distinct DOEs with different output diffraction angles (DOE spatial periods) as explained in the experimental section.

In such conditions, Fig. 6 establishes the superiority of the $100 \times$ objective, with the highest NA, in view of minimizing proximity effects at a given spot separation. Here the temporal component of the proximity effect is also clearly shown with the $40 \times$ objective: the height of each "L" manufactured with this objective is not constant but increases continuously during the manufacturing process (the "L" motifs being fabricated from top left to bottom right as indicated by a red arrow). The beginning of the $\mathrm{L}$ structure is not visible, while its end shows clear signs of over-polymerization. This indicates that as manufacturing progresses, the resin locally becomes artificially more sensitive due to prolonged exposure to the laser beams, consistent with our working-hypothesis of a time-dependant diffusion phenomenon of the generated radicals. In the present case, this effect combines with the overlapping of the light beams, strongly conditioned by the nature of the objective. A slight temporal proximity effect can still be noticed with the $100 \times$ objective, but in a more moderate way given the greater NA resulting in a better confinement of the laser intensity along the $\mathrm{Z}$ axis. As a consequence, it was possible to find a set of parameters leading to globally uniform structures: each individual L-shaped structure shows a relatively homogeneous width and height along the fabrication path.

This process was simulated digitally, translating the $5 \times 5$ PSF write spot array between each laser pulse to model the laser beam scanning with arrays of two different PSF to represent the $100 \times$ NA 1.40 (Fig. 7A) and the 40× NA 0.95 (Fig. 7B) objectives. A satisfactory agreement with the experimentally observed structures was again obtained, limited to some extent by the coarse simulation grid $(200 \mathrm{~nm})$ imposed by the heavy simulation load. We simulated with a number of pulses corresponding to $200 \mathrm{~nm}$ steps at the considered scan rate $\left(2.4 \mu \mathrm{m} \cdot \mathrm{s}^{-1}\right.$ here), meaning about 975 pulses par sampling grid position. As before, the simulated diffusion kernel $\sigma$ value and the decay factor were adapted empirically to fit the simulations to the experimentally observed structures. This resulted in the parameters shown in Table S1 indicating a stronger diffusion behaviour in the PR_hybrid resist. A decay factor of 0.99999 gave the best fit, corresponding to a diffusing species half-life of $5.9 \mathrm{~s}$, which seems again in good agreement with previously reported values for radical species, although this lifetime is longer than that calculated in PR_organic for reasons that will be discussed later.
A)

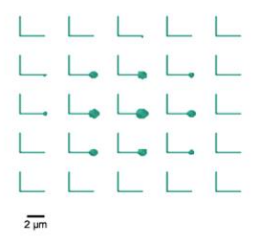

$45^{\circ}$

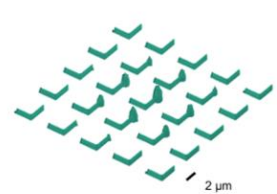

B)

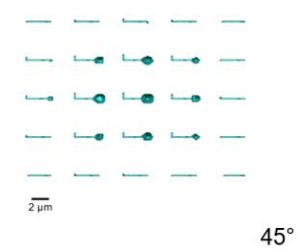

$45^{\circ}$

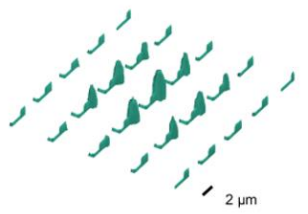

Fig. 7. Results of the digital simulations of the scanned spot array write process showing the influence of the objective NA on the uniformity of $5 \times 5$ "L" networks. (A) NA 0.95, (B) NA 1.40. Top (top) and $45^{\circ}$ (bottom) views are shown for each structure. 
Calculations hence confirm that stronger focusing of the light results in a reduced overlap of the high intensity part of the light beams with the higher NA objective for a given inter-spot distance. As expected, the use of higher NA objectives helps to reduce the spatial extent of the PSF, making this technique a way to minimize overlap and thus reduce specific proximity effects in parallel TPP. Interestingly, in the case of these L-shaped structures, the simulations seem to reproduce not only the spatial component (decreased polymerization threshold from the periphery to the center) but also the temporal component (decreasing polymerization threshold with increasing manufacturing time as indicated by the larger and higher structures written towards the end of the plot) of the proximity effect, thereby confirming the probably crucial role of species diffusion in the overall process.

\subsection{Influence of the photoresist composition}

The spatial resolution of a photopolymerization reaction is not only influenced by the dimensions of the irradiated voxels, but ultimately also by how far growing radicals species can diffuse away from the irradiated area. The latter parameter can be influenced by multiple factors, such as radical intrinsic mobility and the presence and diffusion ability of radical quenchers, such as for instance molecular oxygen or polymerization inhibitors. Both processes are strongly dependent on the nature and composition of the photoresist.

A huge impact of the photoresist composition was indeed highlighted by the comparison between an organic resin PR_organic based on a mixture of acrylates (DPPHA and DDA) and a hybrid resin PR_hybrid based on a modified Ormocomp ${ }^{\circledR}$ resist. The same photoinitiator (PI) was used in both resins so that the difference lies in the monomer mixture composition (organic acrylate mixture stabilized with MEHQ on the one hand, unstabilized hybrid resin on the other). As illustrated in Fig. 8 the PR_hybrid is much more reactive than the PR_organic. Thus, fabrication of a development-resistant structure can be achieved with shorter exposure times in the PR_hybrid resist. However, with the unstabilized PR_hybrid resist, it was impossible to control the polymerization features in order to obtain an homogeneous periodic structure: a marked difference was systematically seen when comparing the central and the peripheral spots, with a clear overpolymerization in the center compared to the corners, regardless of the fabricated test structure (1D: single voxel; 2D: L-shape; 3D: cones).

Conversely, it was possible to find suitable manufacturing parameters to fabricate a homogeneous

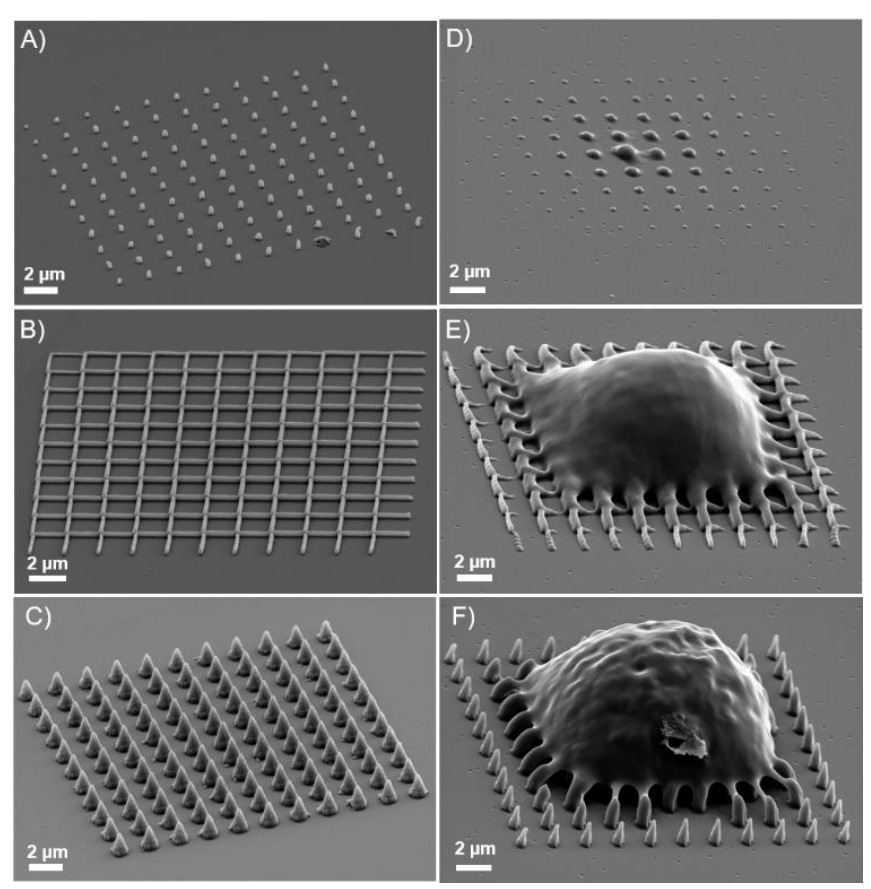

Fig. 8. Effect of resin monomer on the written structures: voxel structure arrays (top), "L" shapes (center) and cones arrays (bottom) manufactured with an $11 \times 11$ spot DOE. Left: PR_organic resin, spacing: $1.85 \mu \mathrm{m}$. $P_{\text {total }}=5.4 \mathrm{~mW}, 100 \times$ objective, exposure time: (A) $5 \mathrm{~s}$ and scanning speeds: (B) 120 $\mathrm{nm} \cdot \mathrm{s}^{-1}$ and (C) $240 \mathrm{~nm} \cdot \mathrm{s}^{-1}$. Right: PR_hybrid, spacing: $1.85 \mu \mathrm{m}$. $P_{\text {total }}=5.4 \mathrm{~mW}, 100 \times$ objective, exposure time: (D) $50 \mathrm{~ms}$ and scanning speeds: (E) $40 \mu \mathrm{m} . \mathrm{s}^{-1}$ and, (F) $120 \mu \mathrm{m} . \mathrm{s}^{-1}$.

network of the three selected test structures with the PR_organic resin (a single voxel, an "L" shape with a side length of $2 \mu \mathrm{m}$ and a cone with a height of $1.40 \mu \mathrm{m}$ ) at an inter-spot distance as small as $1.85 \mu \mathrm{m}$.

Fig. 9 shows the corresponding simulation results. Good empirical fits to the experimental voxel structures (illustrations A and D in Fig. 8 and Fig. 9) were obtained using the appropriate number of laser pulses, 58500 and 585 laser pulses for PR_organic and PR_hybrid respectively, and the simulation parameters used previously for both resists (see Table S1).

In the case of the "L" shapes simulations (illustrations $B$ and $E$ in Fig. 8 and Fig. 9), the same diffusion parameters gave the best experimental fit, considering 19500 and 59 pulses per sample grid position every 200 $\mathrm{nm}$ for PR_organic and PR_hybrid respectively, in accordance with the scan speed.

These results seem to indicate that diffusion in the PR_hybrid resist is significantly stronger than in the PR_organic resist, being of both longer range (higher $\sigma$ value) and with a longer diffusing species lifetime (lower decay rate). Besides, Fig. 9B shows significant out of focal plane polymerization which also appears in the simulations but to a more limited extent, probably 
because the simulations only model interactions close to the focus plane and do not take out of plane light energy into effect (computational load limitations currently prevent this). As explained above (Fig. 5 and Fig. S3), such out of focus plane interactions very probably do occur (particularly for large arrays such as the $11 \times 11$ spot array used here) and are likely to be at least partially responsible for the increased height of the experimental structures of the PR_hybrid structures.

Because of the unstabilized nature of PR_hybrid and the wide range of evidence obtained in all of the abovedescribed experiments for the involvement of diffusion (probably of radical species) in the proximity effects and most notably the much increased lifetime of the diffusing species in PR-hybrid resist compared to PR_organic, we hypothesized that addition of MEHQ as stabilizer should help in reducing proximity effects in the latter. Thus, a stabilized equivalent of PR_hybrid, where $700 \mathrm{ppm}$ MEHQ were added to the formulation was prepared and used for parallel microfabrication with an $11 \times 11$ spot DOE at $1.85 \mu \mathrm{m}$ inter-spot distance. Systematic comparison between results obtained with PR_hybrid and PR_hybrid stabilized with 700 ppm MEHQ (Fig. S4) clearly establishes that the addition of the stabilizer consistently reduces proximity effects (at the expense of reduced photoresist sensitivity). As a consequence, the quality of periodic arrays fabricated with PR_hybrid with 700 ppm MEHQ becomes relatively similar to that obtained with PR_organic, although a slight central over-polymerization was still observed.
A)

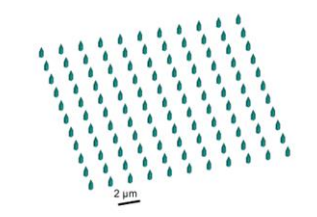

B)

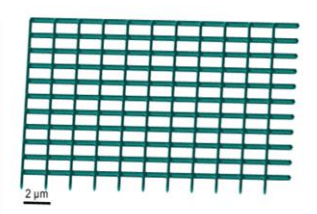

D)

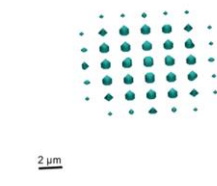

E)

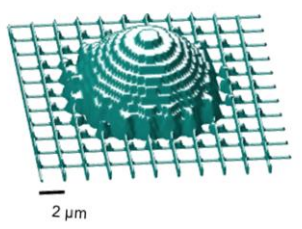

Fig. 9. Results of the digital simulation of the parallel-write process, showing of the effect of resin monomer properties on the structures obtained for voxel structure arrays (top) and " $L$ " shapes (bottom). (Left: PR_organic resin, Right: PR_hybrid). Cones were not simulated due to computational load.

\subsection{Overcoming proximity effects}

In the above, we have seen that spatio-temporal proximity effects in parallel writing appear to be caused or reinforced by different factors: small inter-spot distance of the DOE generated write spot array, low numerical aperture of the objective and chemical nature of the photoresist (monomer mixture). All these parameters have in common that they render the polymerization threshold in the vicinity of the exposed areas more sensitive to radical diffusion effects. Moreover, at very short (sub-micron) inter-voxel distances, other optical effects, conceptually related to the well-known Talbot effect, also appear to come into play and hamper parallel writing.

With all these considerations in mind, we compared different writing procedures for the fabrication of a periodically structured surface featuring 900 voxel structures in a $30 \times 30$ array with an inter-structure distance of $0.9 \mu \mathrm{m}$ : at such separations, we have shown that all proximity effects were systematically exacerbated.

Thus, using an objective with a numerical aperture of 1.40 , we designed three distinct writing procedures (Fig. 10, left column). In order to keep relatively short exposure times $(10 \mathrm{~ms})$, the more reactive Ormocomp based resist was preferred, in spite of its higher sensitivity to proximity effects as demonstrated above.

A/ In a first procedure, a $5 \times 5$ DOE generated spot pattern with a low inter-spot separation, $\mathrm{p}=0.9 \mu \mathrm{m}$, was translated with a long step distance, $\mathrm{D}=4.4 \mu \mathrm{m}$, to generate the expected 900 voxel structure array by 36 successive translations and exposures $(6 \times 6$ periodic square motif), with an overall fabrication time of $22.5 \mathrm{~s}$.

$\mathrm{B}$ / In a second procedure, the $5 \times 5$ spot array featured a greater inter-spot separation of $\mathrm{D}=5.5 \mu \mathrm{m}$; thus, in this case, the 900 voxel array was generated using 36 translations with short step of $\mathrm{p}=0.9 \mu \mathrm{m}$ and exposures. The total fabrication time in this case was also $22.5 \mathrm{~s}$.

C/ Finally, as a reference, the same structure was generated by single beam point-by-point writing (900 translations of $0.9 \mu \mathrm{m}$ and subsequent exposures), in a $30 \times 30$ square array motif. In this case, the fabrication was achieved in 558.2 seconds.

Fabrication conditions, translation pattern and fabrication outcome as visualized by SEM are gathered in Fig. 10. 


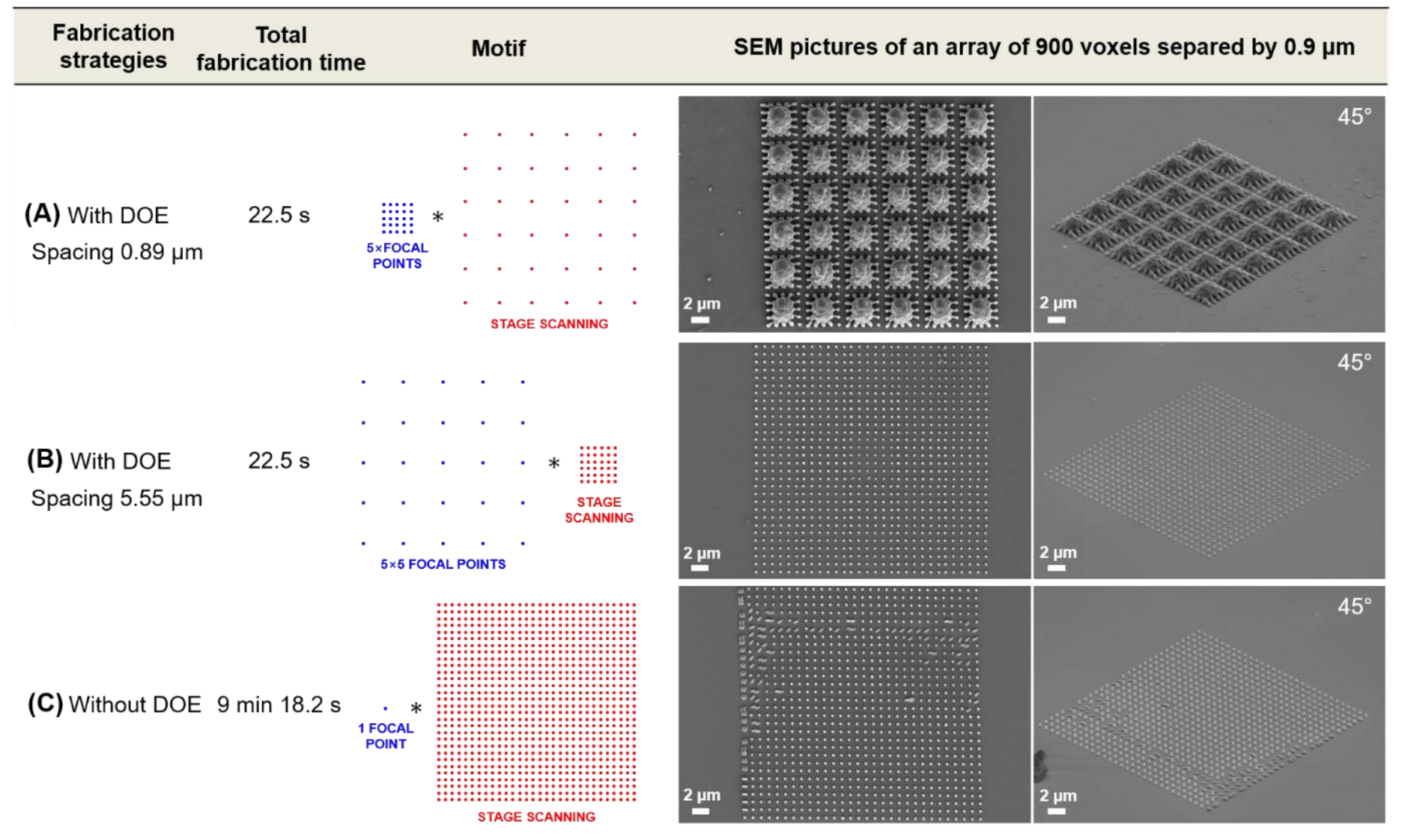

Fig. 10 Comparison of two parallel and one non-parallel printing strategies for the fabrication of a $900(30 \times 30)$ voxel structure array with an inter-structure distance of $c a .0 .9 \mu \mathrm{m}$ : (A) with a DOE spot array period of 0.9 $\mu \mathrm{m}$, (B) with a DOE spot array period of $5.55 \mu \mathrm{m}$, (C) without a DOE, point by point writing (reference). Objective 100x, Photoresist: PR_hybrid. Exposure time for each individual voxel structure: $10 \mathrm{~ms}$. $(\mathrm{A}) \mathbf{P}_{\text {total }}=$ $4.4 \mathrm{~mW}$; (B) $P_{\text {total }}=4.4 \mathrm{~mW}$; (C) $P=123 \mu W$.

The results obtained clearly illustrate the benefits of using strategies involving larger spot separation DOEs and shorter translation distances in the parallel fabrication of small (sub-micron) step distance periodic arrays of motifs. While the use of a short inter-spot separation DOE results in marked proximity effects that strongly affect the quality and homogeneity of the periodic structure (Fig. 10A), these effects are completely absent with larger inter-spot distance patterns (Fig. 10B).

Thus, the result is comparable with that obtained using single beam point-by-point printing (Fig. 10C) but with a write duration 25 times shorter (22.5 s vs $558.2 \mathrm{~s})$.

\section{Conclusions}

While already identified and reported in the past for serial single-spot multi-photon DLW, spatio-temporal proximity effects appear particularly critical when parallel fabrication is involved, especially when short inter-structure distances are targeted. The combined experimental data and simulations described above allow us to identify some key experimental parameters that determine the outcome of parallel two-photon DLW printing in our mechanistic study.

These data illustrate that, when parallelizing twophoton DLW using DOEs, many criteria have to be taken into account to ensure maximal efficiency of the fabrication process and fidelity in the reproduction of the pattern array.

In particular, we have shown that laser power, the NA of the objective, the chemical nature of the resin and, most importantly, the inter-spot distance of the projected motifs are particularly determining factors that have a strong influence on the strength of these proximity effects. Attempts to model these effects through digital simulations revealed a predominant influence of diffusion processes on the characteristics of the intensity of these proximity effects. More precisely, the characteristic diffusion length and time dependence of the diffusion phenomenon led us to formulate the hypothesis of short lived radicals being the diffusing species. As a support to this hypothesis, we showed that the introduction of ppm amounts of a free radical inhibitor (MEHQ) into the hybrid Ormocomp photoresist, in which proximity effects were particularly strong, led to a marked reduction of the range of these effects. 
With these considerations in mind, we have proposed a plot strategy that enables these proximity effects to be largely circumvented. This strategy consists in generating patterns with a targeted small inter-structure distances through small displacements of a large interspot spacing DOE generated spot arrays. Despite the limited beam density in the fabrication area, imposing a compromise between DLW throughput and proximity effects, the proposed strategy represents an important step towards massively parallelized high-resolution additive fabrication. We have clearly illustrated that our strategy is efficient in bypassing proximity effects and thus in obtaining sub-micron resolution structures with high reproduction fidelity and greatly reduced fabrication times compared to the single beam approach.

Further studies would be interesting to extend the range of fabrication speeds, to fully understand the proximity effects in multi-photon DLW and determine the optimal writing speed ranges to completely suppress or at least control proximity effects. In particular, larger proximity effects have been reported for example when increasing the scanning speed for single-beam fabrication at significantly higher speeds $\left(50 \mathrm{~mm} \cdot \mathrm{s}^{-1}\right)$ [15] than used in our study.

We believe that the results presented in this paper and the consequent fabrication strategy will inspire further experimental and theoretical work in the parallel microfabrication of periodic structures with sub-micron motif separations.

\section{Acknowledgments}

The authors thank Fabian Hilbert (Multiphoton Optics $\mathrm{GmbH}$ ) and Ruth Houbertz (ThinkMade Engineering \& Consulting i.Gr.) for inspiring discussions.

\section{Funding}

ENS Lyon authors acknowledge the Agence Nationale de la Recherche ANR for the grant "New 3D print", especially C.A for doctoral grant.

This work has received funding from the European Union's Horizon 2020 research and innovation program under grant agreement $\mathrm{N}^{\circ} 780278$. The dissemination of results herein reflects only the author's view and the European Commission is not responsible for any use that may be made of the information it contains.

\section{References}

[1] S.C. Ligon, R. Liska, J. Stampfl, M. Gurr, R. Mülhaupt, Polymers for 3D Printing and Customized Additive Manufacturing, Chem. Rev. 117 (2017) 10212-10290. https://doi.org/10.1021/acs.chemrev.7b00074.
[2] S. Maruo, J.T. Fourkas, Recent progress in multiphoton microfabrication, Laser Photonics Rev. 2 (2008) 100-111. https://doi.org/10.1002/lpor.200710039.

[3] C. Barner-Kowollik, M. Bastmeyer, E. Blasco, G. Delaittre, P. Müller, B. Richter, M. Wegener, 3D Laser Micro- and Nanoprinting: Challenges for Chemistry, Angew. Chem. Int. Ed. 56 (2017) 15828-15845. https://doi.org/10.1002/anie.201704695.

[4] J. Kato, N. Takeyasu, Y. Adachi, H.-B. Sun, S. Kawata, Multiple-spot parallel processing for laser micronanofabrication, Appl. Phys. Lett. 86 (2005)

044102 . https://doi.org/10.1063/1.1855404.

[5] C.S. Lim, M.H. Hong, Y. Lin, Q. Xie, B.S. Luk'yanchuk, A. Senthil Kumar, M. Rahman, Microlens array fabrication by laser interference lithography for super-resolution surface nanopatterning, Appl. Phys. Lett. 89 (2006) 191125. https://doi.org/10.1063/1.2374809.

[6] H. Lin, B. Jia, M. Gu, Dynamic generation of Debye diffraction-limited multifocal arrays for direct laser printing nanofabrication, Opt. Lett. 36 (2011) 406-408. https://doi.org/10.1364/OL.36.000406.

[7] H. Ren, H. Lin, X. Li, M. Gu, Three-dimensional parallel recording with a Debye diffractionlimited and aberration-free volumetric multifocal array, Opt. Lett. 39 (2014) 1621-1624. https://doi.org/10.1364/OL.39.001621.

[8] P.S. Salter, M.J. Booth, Addressable microlens array for parallel laser microfabrication, Opt. Lett. 36 (2011) 2302-2304. https://doi.org/10.1364/OL.36.002302.

[9] K. Obata, J. Koch, U. Hinze, B.N. Chichkov, Multi-focus two-photon polymerization technique based on individually controlled phase modulation, Opt. Express. 18 (2010) 1719317200. https://doi.org/10.1364/OE.18.017193.

[10] S.D. Gittard, A. Nguyen, K. Obata, A. Koroleva, R.J. Narayan, B.N. Chichkov, Fabrication of microscale medical devices by two-photon polymerization with multiple foci via a spatial light modulator, Biomed. Opt. Express. 2 (2011) 3167-3178. https://doi.org/10.1364/BOE.2.003167.

[11] G. Vizsnyiczai, L. Kelemen, P. Ormos, Holographic multi-focus 3D two-photon polymerization with real-time calculated holograms, Opt. Express. 22 (2014) 24217 24223. https://doi.org/10.1364/OE.22.024217.

[12] Q. Geng, D. Wang, P. Chen, S.-C. Chen, Ultrafast multi-focus 3-D nano-fabrication based on twophoton polymerization, Nat. Commun. 10 (2019) 2179. https://doi.org/10.1038/s41467-019-102492.

[13] V. Hahn, P. Kiefer, T. Frenzel, J. Qu, E. Blasco, C. Barner-Kowollik, M. Wegener, Rapid Assembly of Small Materials Building Blocks 
(Voxels) into Large Functional 3D Metamaterials, Adv. Funct. Mater. 30 (2020) 1907795. https://doi.org/10.1002/adfm.201907795.

[14] L. Zheng, K. Kurselis, A. El-Tamer, U. Hinze, C. Reinhardt, L. Overmeyer, B. Chichkov, Nanofabrication of High-Resolution Periodic Structures with a Gap Size Below $100 \mathrm{~nm}$ by Two-Photon Polymerization, Nanoscale Res. Lett. 14 (2019) 134. https://doi.org/10.1186/s11671019-2955-5.

[15] J.S. Oakdale, R.F. Smith, J.-B. Forien, W.L. Smith, S.J. Ali, L.B.B. Aji, T.M. Willey, J. Ye, A.W. van Buuren, M.A. Worthington, S.T. Prisbrey, H.-S. Park, P.A. Amendt, T.F. Baumann, J. Biener, Direct Laser Writing of LowDensity Interdigitated Foams for Plasma Drive Shaping, Adv. Funct. Mater. 27 (2017) 1702425. https://doi.org/10.1002/adfm.201702425.

[16] E. Waller, G. von Freymann, Spatio-Temporal Proximity Characteristics in 3D $\mu$-Printing via Multi-Photon Absorption, Polymers. 8 (2016) 297. https://doi.org/10.3390/polym8080297.

[17] S.H. Park, T.W. Lim, D.-Y. Yang, N.C. Cho, K.S. Lee, Fabrication of a bunch of sub-30-nm nanofibers inside microchannels using photopolymerization via a long exposure technique, Appl. Phys. Lett. 89 (2006) 173133. https://doi.org/10.1063/1.2363956.

[18] D. Tan, Y. Li, F. Qi, H. Yang, Q. Gong, X. Dong, $\mathrm{X}$. Duan, Reduction in feature size of two-photon polymerization using SCR500, Appl. Phys. Lett. $90 \quad$ (2007) 071106. https://doi.org/10.1063/1.2535504.

[19] Y. Bougdid, I. Maouli, A. Rahmouni, K. Mochizuki, I. Bennani, M. Halim, Z. Sekkat, Systematic lambda/21 resolution achieved in nanofabrication by two-photon-absorption induced polymerization, J. Micromech. Microeng. 29 (2019) 035018. https://doi.org/10.1088/13616439/aafda0.

[20] J.B. Mueller, J. Fischer, F. Mayer, M. Kadic, M. Wegener, Polymerization Kinetics in ThreeDimensional Direct Laser Writing, Adv. Mater. 26 (2014) 6566-6571. https://doi.org/10.1002/adma.201402366.

[21] L. Yang, A. Münchinger, M. Kadic, V. Hahn, F. Mayer, E. Blasco, C. Barner-Kowollik, M. Wegener, On the Schwarzschild Effect in 3D Two-Photon Laser Lithography, Adv. Opt. Mater. $7 \quad$ (2019) 1901040. https://doi.org/10.1002/adom.201901040.

[22] X. Wan, R. Menon, Proximity-effect correction for 3D single-photon optical lithography, Appl. Opt. $55 \quad$ (2016) A1-A7. https://doi.org/10.1364/AO.55.0000A1.

[23] M.T. Do, T.T.N. Nguyen, Q. Li, H. Benisty, I. Ledoux-Rak, N.D. Lai, Submicrometer 3D structures fabrication enabled by one-photon absorption direct laser writing, Opt. Express. 21
(2013)

20964-20973.

https://doi.org/10.1364/OE.21.020964.

[24] S. Rodríguez, Redefining Microfabrication of High-Precision Optics, PhotonicsViews. 17 (2020) 36-39. https://doi.org/10.1002/phvs.202000003.

[25] L. Jiang, W. Xiong, Y. Zhou, Y. Liu, X. Huang, D. Li, T. Baldacchini, L. Jiang, Y. Lu, Performance comparison of acrylic and thiolacrylic resins in two-photon polymerization, Opt. Express. 24 (2016) 13687-13701. https://doi.org/10.1364/OE.24.013687.

[26] S.K. Saha, C. Divin, J.A. Cuadra, R.M. Panas, Effect of Proximity of Features on the Damage Threshold During Submicron Additive Manufacturing Via Two-Photon Polymerization, J Micro Nanomanuf. 5 (2017) 031002. https://doi.org/10.1115/1.4036445.

[27] S.K. Saha, D. Wang, V.H. Nguyen, Y. Chang, J.S. Oakdale, S.-C. Chen, Scalable submicrometer additive manufacturing, Science. 366 (2019) 105109. https://doi.org/10.1126/science.aax8760.

[28] O. Ripoll, V. Kettunen, H.P. Herzig, Review of iterative Fourier-transform algorithms for beam shaping applications, Opt. Express. 43 (2004) 2549-2548. https://doi.org/10.1117/1.1804543.

[29] F. Wyrowski, Iterative quantization of digital amplitude holograms, Appl. Opt., AO. 28 (1989) 3864-3870. https://doi.org/10.1364/AO.28.003864.

[30] M.M. Kessels, M.E. Bouz, R. Pagan, K.J. Heggarty, Versatile stepper based maskless microlithography using a liquid crystal display for direct write of binary and multilevel microstructures, J Micro Nanolithogr MEMS MOEMS. 6033002. https://doi.org/10.1117/1.2767331.

[31] M.V. Kessels, C. Nassour, P. Grosso, K. Heggarty, Direct write of optical diffractive elements and planar waveguides with a digital micromirror device based UV photoplotter, Opt. Commun. $283 \quad$ (2010) 3089-3094. https://doi.org/10.1016/j.optcom.2010.03.056.

[32] C. Arnoux, T. Konishi, E. Van Elslande, E.-A. Poutougnigni, J.-C. Mulatier, L. Khrouz, C. Bucher, E. Dumont, K. Kamada, C. Andraud, P. Baldeck, A. Banyasz, C. Monnereau, Polymerization Photoinitiators with NearResonance Enhanced Two-Photon Absorption Cross-Section: Toward High-Resolution Photoresist with Improved Sensitivity, Macromolecules. 53 (2020) 9264-9278. https://doi.org/10.1021/acs.macromol.0c01518.

[33] J. Rouillon, C. Arnoux, C. Monnereau, Determination of Photoinduced Radical Generation Quantum Efficiencies by Combining Chemical Actinometry and 19F NMR Spectroscopy, Anal. Chem. 93 (2021) 2926-2932. https://doi.org/10.1021/acs.analchem.0c04540. 
[34] $\mathrm{BIG} \cdot \mathrm{PSF}$ Generator, http://bigwww.epfl.ch/algorithms/psfgenerator/ (accessed April 29, 2021).

[35] F. Hilbert, J. Wiedenmann, B. Stender, W. Mantei, R. Houbertz, Q. Carlier, L.P. Covarrubias, K. Heggarty, C. Arnoux, C. Monnereau, P. Baldeck, Impact of massive parallelization on two-photon absorption microand nanofabrication, Proc. SPIE 11271, Laser 3D Manufacturing VII (2020) 1127105. https://doi.org/10.1117/12.2545788.

[36] D. Griller, K.U. Ingold, Persistent carboncentered radicals, Acc. Chem. Res. 9 (1976) 1319. https://doi.org/10.1021/ar50097a003.

[37] J. Wen, Y. Zhang, M. Xiao, The Talbot effect: recent advances in classical optics, nonlinear optics, and quantum optics, Adv. Opt. Photon. 5 (2013)

83-130.
[38] H.H. Solak, C. Dais, F. Clube, Displacement Talbot lithography: a new method for highresolution patterning of large areas, Opt. Express. 19 10686-10691. https://doi.org/10.1364/OE.19.010686.

[39] P.-M. Coulon, B. Damilano, B. Alloing, P. Chausse, S. Walde, J. Enslin, R. Armstrong, S. Vézian, S. Hagedorn, T. Wernicke, J. Massies, J. Zúñiga-Pérez, M. Weyers, M. Kneissl, P.A. Shields, Displacement Talbot lithography for nano-engineering of III-nitride materials, Microsyst. Nanoeng. 5 (2019) 1-12. https://doi.org/10.1038/s41378-019-0101-2.

[40] D.J. Shir, S. Jeon, H. Liao, M. Highland, D.G. Cahill, M.F. Su, I.F. El-Kady, C.G. Christodoulou, G.R. Bogart, A.V. Hamza, J.A. Rogers, Three-Dimensional Nanofabrication with Elastomeric Phase Masks, J. Phys. Chem. B. 111 (2007) 12945-12958. https://doi.org/10.1021/jp074093j. 